\title{
Synthetic inhibitors of CDKs induce different responses in androgen sensitive and androgen insensitive prostatic cancer cell lines
}

\author{
J Mad'arová, M Lukešová, A Hlobilková, M Strnad, B Vojtěšek, R Lenobel, M Hajdúch, \\ P G Murray, S Perera, Z Kolář
}

J Clin Pathol: Mol Pathol 2002;55:227-234

See end of article for authors' affiliations .....................

Correspondence to: Dr Z Kolář, Laboratory of Molecular Pathology, Faculty of Medicine, Centre of Molecular Biology and Medicine, Palacký University, Hněvotínská 3 , CZ-77515 Olomouc, Czech Republic; kolarz@tunw.upol.cz

Accepted for publication 4 December 2001

\begin{abstract}
Aims: Because of the high prevalence of prostatic cancer and the limitations of its treatment, enormous effort has been put into the development of new therapeutic modalities. One potential tool is the use of cyclin dependent kinase (CDK) inhibitors, which are based on the trisubstituted derivatives of purine. The aim of this study was to analyse alterations of the regulatory pathways in both androgen sensitive and androgen insensitive prostatic cancer cell lines (LNCaP and DU-145, respectively) after blockage of the cell cycle by the synthetic CDK inhibitors, olomoucine and bohemine.

Methods: The effects of olomoucine and bohemine were studied on the following parameters: (1) cell proliferation, by measurement of DNA content; (2) viability, by the MTT (3-[4,5-dimethylthiazol-2-yl]2,5-diphenyltetrazolium bromide) and/or XTT (2,3-bis[2-methoxy-4-nitro-5-sulfophenyl]-2H-tetrazolium5-carboxanilide) test; and (3) the expression of p53, pRB, Bcl-2, Bax, p16, p2 1, p27, cyclins A, B, D1, $\mathrm{E}, \mathrm{p} 34^{\mathrm{cdc} 2}$, and the androgen receptor (AR), by western blot analysis.

Results: Both olomoucine and bohemine were potent inhibitors of growth and viability; however, bohemine was two to three times more effective than olomoucine. The sensitivity of LNCaP cells to both agents was significantly higher. After treatment, both cell lines revealed quite different spectra of protein expression.

Conclusions: These results indicate the existence of specific cell cycle regulating pathways in both cell lines, which may be associated with both p53 and AR status. CDK inhibitors exhibited valuable secondary effects on the expression of numerous regulators and thus may modulate the responsiveness of tumour cells to treatment, including treatment with hormone antagonists.
\end{abstract}

B ecause of the high prevalence of prostatic cancer and the limitations of its treatment, enormous effort has been put into the development of new therapeutic modalities. One potential tool is the use of cyclin dependent kinase (CDK) inhibitors, which are based on the trisubstituted derivatives of purine. $^{12}$ The potential therapeutic effects of olomoucine (2-[2-hydroxyethylamino]-6-benzylamino-9-methylpurine) and its analogue bohemine (2-[3-hydroxypropylamino]-6benzylamino-9-isopropylpurine) on various cancer cell lines have been described. ${ }^{34}$

Steroid hormones and growth factors are involved in the regulation of cell proliferation and apoptosis in hormone sensitive prostatic tumours. ${ }^{56}$ Numerous changes in the expression of cell cycle and apoptosis regulating proteins have been described during the development of hormone insensitive prostatic cancer. ${ }^{6-9}$ Probably most attention has been focused on the relation between androgen receptor (AR) expression and its ability to regulate the proliferation and expression of downstream proteins. ${ }^{6}{ }^{10}$ However, little information is available on the relation between upstream regulators of $\mathrm{AR}$ expression and AR function.

There are several regulators in the AR signalling pathway, including the tumour suppressor genes, p53 and retinoblastoma (RB); the apoptosis related genes, bcl-2 and bax; and the endogenous inhibitors of the CDKs, p16, p21, and p27..$^{6-8}$ This prompted questions concerning cooperation between these factors in the course of cell cycle arrest. The unique effects of olomoucine and bohemine make it possible to analyse these changes and these agents provide an excellent tool to study such relations within the cell cycle. In our present study, we analysed the effect of these cell death inducing agents on cells with the typical characteristics of either hormone responsive or hormone refractory prostatic cancer; that is, cell lines with androgen responsive but mutated ARs (LNCaP) and androgen unresponsive but wild-type ARs (DU-145).

"Numerous changes in the expression of cell cycle and apoptosis regulating proteins have been described during the development of hormone insensitive prostatic cancer"

After treatment with olomoucine and bohemine we found induction of AR in DU-145 cells but not in LNCaP cells, and there were significant differences in the expression of upstream and downstream proteins between the cell lines. Thus, the expression of p53, Bax, p21, all tested cyclins, and p34 ${ }^{\text {cdc2 }}$ decreased in the androgen insensitive DU-145 cells within 72 hours of exposure to a death inducing agent, whereas the expression of p27, pRB, and p16 increased. However, in LNCaP cells, which have the wild-type p53 gene, we recorded an increase in both p53 and p21 within 24 hours of treatment and a general decrease in Bcl-2 and AR within 24 to 72 hours. Furthermore, we also noted an increase in the expression of cyclin D1, cyclin E, and p27 but decreased expression of the remaining cyclins and $\mathrm{p} 34^{\mathrm{cdc} 2}$.

Abbreviations: $A R$, androgen receptor; $C D K$, cyclin dependent kinase; MTT, 3-[4,5-dimethylthiazol-2-yl]-2,5-diphenyltetrazolium bromide; PBS-T, Tween 20 in phosphate buffered saline; SDS, sodium dodecyl sulfate; PRB, retinoblastoma protein; XTT, 2,3-bis[2-methoxy-4-nitro-5-sulfophenyl]$2 \mathrm{H}$-tetrazolium-5-carboxanilide 

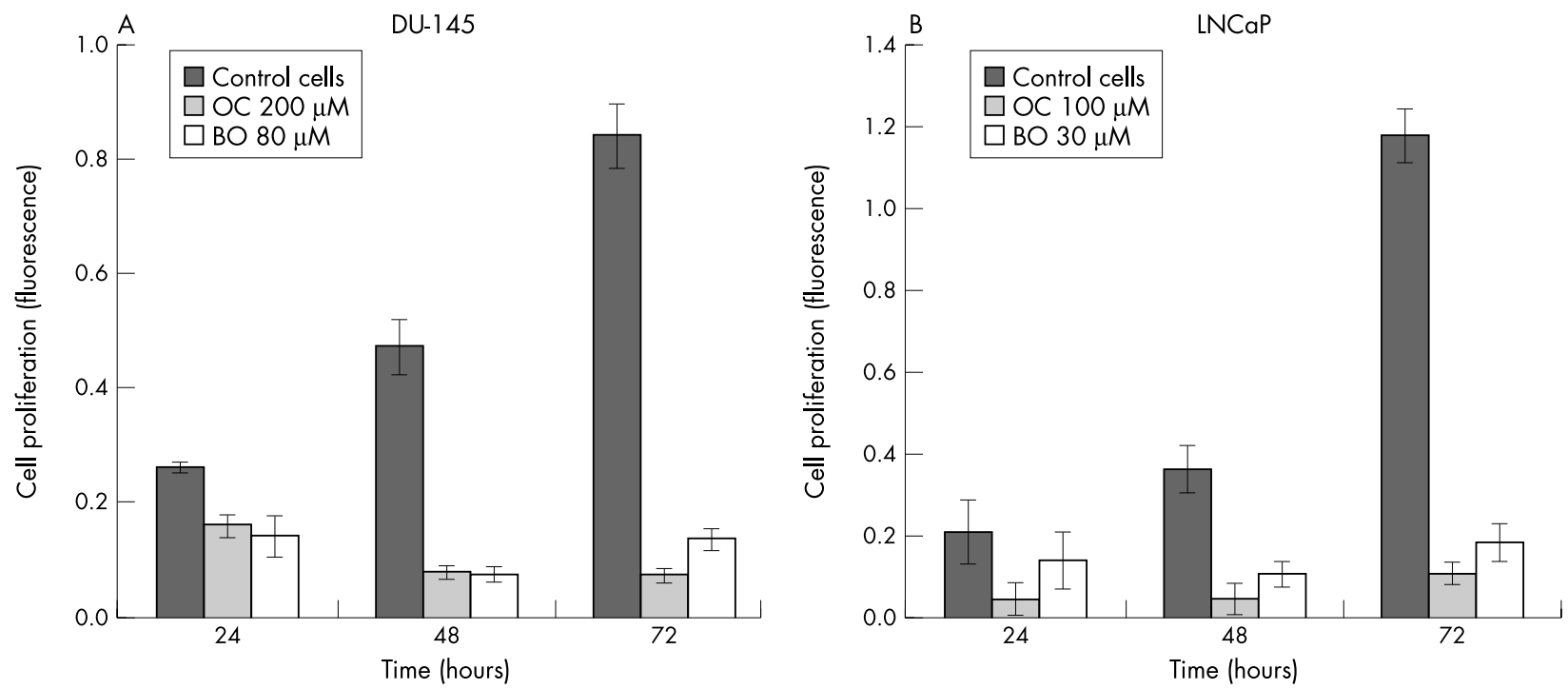

Figure 1 The effect of $\approx \mathrm{I}_{50}$ concentrations of olomoucine $(\mathrm{OC})$ and bohemine $(\mathrm{BO})$ on cell proliferation measured by Hoechst dye staining

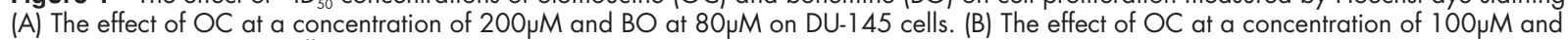
$\mathrm{BO}$ at $30 \mu \mathrm{M}$ on $\mathrm{LNCaP}$ cells.

\section{MATERIALS AND METHODS Cell culture}

The LNCaP cell line was obtained from the American Type Culture Collection (Rockville, Maryland, USA) and the DU-145 cell line was kindly provided by Dr J Bartek (Danish Cancer Society, Copenhagen, Denmark). LNCaP cells were cultured in RPMI 1640 medium (Sigma, St Louis, Missouri, USA), supplemented with $10 \%$ fetal calf serum and L-glutamine $(2 \mathrm{mM})$, and containing sodium bicarbonate ( $1.5 \mathrm{~g} /$ litre), glucose ( $4.5 \mathrm{~g} /$ litre), HEPES (10mM), penicillin $(20000 \mathrm{U} / \mathrm{ml})$, and streptomycin $(100 \mu \mathrm{g} / \mathrm{ml})$. DU-145 cells were grown in Dulbecco's modified Eagles's medium (DMEM; Sigma) supplemented with $10 \%$ fetal calf serum, L-glutamine (5 mM), penicillin $(20000 \mathrm{U} / \mathrm{ml})$, and streptomycin $(100 \mu \mathrm{g} /$ $\mathrm{ml}$ ). Both cell lines were kept in a humidified atmosphere of $5 \% \mathrm{CO}_{2}$ at $37^{\circ} \mathrm{C}$. Cells were plated on plastic dishes and after reaching $70 \%$ confluence the culture medium was replaced with medium containing olomoucine and/or bohemine to a final concentration of $25-200 \mu \mathrm{M}$ olomoucine or $10-160 \mu \mathrm{M}$ bohemine. The cells were treated for 24,48 , and 72 hours and then cell proliferation and viability were assessed and protein expression was analysed.

\section{Measurement of cell proliferation and viability}

The proliferation and viability of both cell lines in the presence of olomoucine and bohemine were measured by the determination of DNA content and by the MTT (3-[4,5dimethylthiazol-2-yl]-2,5-diphenyltetrazolium bromide) and/or XTT (2,3-bis[2-methoxy-4-nitro-5-sulfophenyl]-2Htetrazolium-5-carboxanilide) dye conversion assays. ${ }^{11-13}$ All manipulations were repeated and performed in triplicate.

\section{Immunoblotting}

Cell lysates were obtained by resuspending cell pellets in sodium dodecyl sulfate (SDS) sample buffer (2\% SDS, $62.5 \mathrm{mM}$ Tris/HCl, pH 6.8, 20\% glycerol, and $0.025 \%$ bromophenol blue). Samples were boiled for four minutes and the protein content was assayed using a modified Lowry method. ${ }^{14} \beta$ Mercaptoethanol was added to each sample to a final concentration of $5 \%$ and equal aliquots of total protein were loaded on to $8 \%, 10 \%$, or $12 \%$ stacked SDS polyacrylamide gels. After electrophoresis, the proteins were electrotransferred to a nitrocellulose membrane (Amersham, Little Chalfont, UK). The blots were blocked with $5 \%$ (wt/vol) nonfat dry milk in PBS-T buffer $(0.1 \%$ Tween 20 in phosphate buffered saline) for at least two hours at room temperature and incubated overnight $\left(4^{\circ} \mathrm{C}\right)$ in an optimally diluted primary antibody (see below). After washing in PBS-T buffer (30 minutes), blots were incubated in secondary peroxidase conjugated antimouse or antirabbit antibody (dilution, 1/4000 and 1/2500, respectively; USOL, Prague, Czech Republic) for 30 minutes at room temperature. Protein bands were visualised by the enhanced chemiluminescent detection system, ECL Plus, according to the protocol provided by the manufacturer (Amersham). The density of bands was evaluated by scanning (imaging densitometer GS-700, software Multi-analyst, version 1.1; BioRad, Hercules, California, USA). Equality of protein loading was confirmed by Ponceau S red (Sigma) staining of the membrane and by immunoblotting for $\alpha$ tubulin (Sigma). The monoclonal antibodies used were: clone 100 (anti-Bcl-2; Biogenex, San Ramon, California, USA), clone F39.4.1 (anti-AR; Biogenex), clone AR44l (anti-AR; Dako, Glostrup, Denmark), clone DCS-50 (anti-pl6; Novocastra, Newcastle upon Tyne, UK), clone 118 (anti-p21; kindly provided by Dr X Lu, Ludwig Institute for Cancer Research, London, UK), clone SX53G8 (anti-p27; Dako), clone HCDCl (anti-p34 ${ }^{\text {ddc2 }}$; Biogenex), clone DO-7 (anti-p53; produced by BV in our laboratories), clone IF8 (anti-pRB; Novocastra), clone 6E6 (anti-cyclin A; Novocastra), clone 7A9 (anti-cyclin B; Novocastra), clone DCS-6 (anti-cyclin D1; Novocastra), clone 13A3 (anti-cyclin E; Novocastra). A polyclonal rabbit antihuman Bax antibody (N-20; Santa Cruz Biotechnology, Santa Cruz, California, USA) was also used. Coloured markers (Rainbow ${ }^{\mathrm{TM}}$; Amersham) were used as protein molecular weight standards.

\section{RESULTS}

Effect of CDK inhibitors on cell proliferation and viability

The CDK inhibitors, olomoucine and bohemine, efficiently induced cell death in both LNCaP and DU- 145 prostate cancer cells. This was demonstrated by either the MTT/XTT test or Hoechst dye staining, which showed a significant decrease in cell viability and a decrease in cell number (figs 1A,B, 2A-D). Similar responses were seen with various concentrations of olomoucine and bohemine, which showed that bohemine is approximately two to three times more effective than olomoucine. For western blot experiments we selected the concentrations of these agents that caused approximately 50\% 

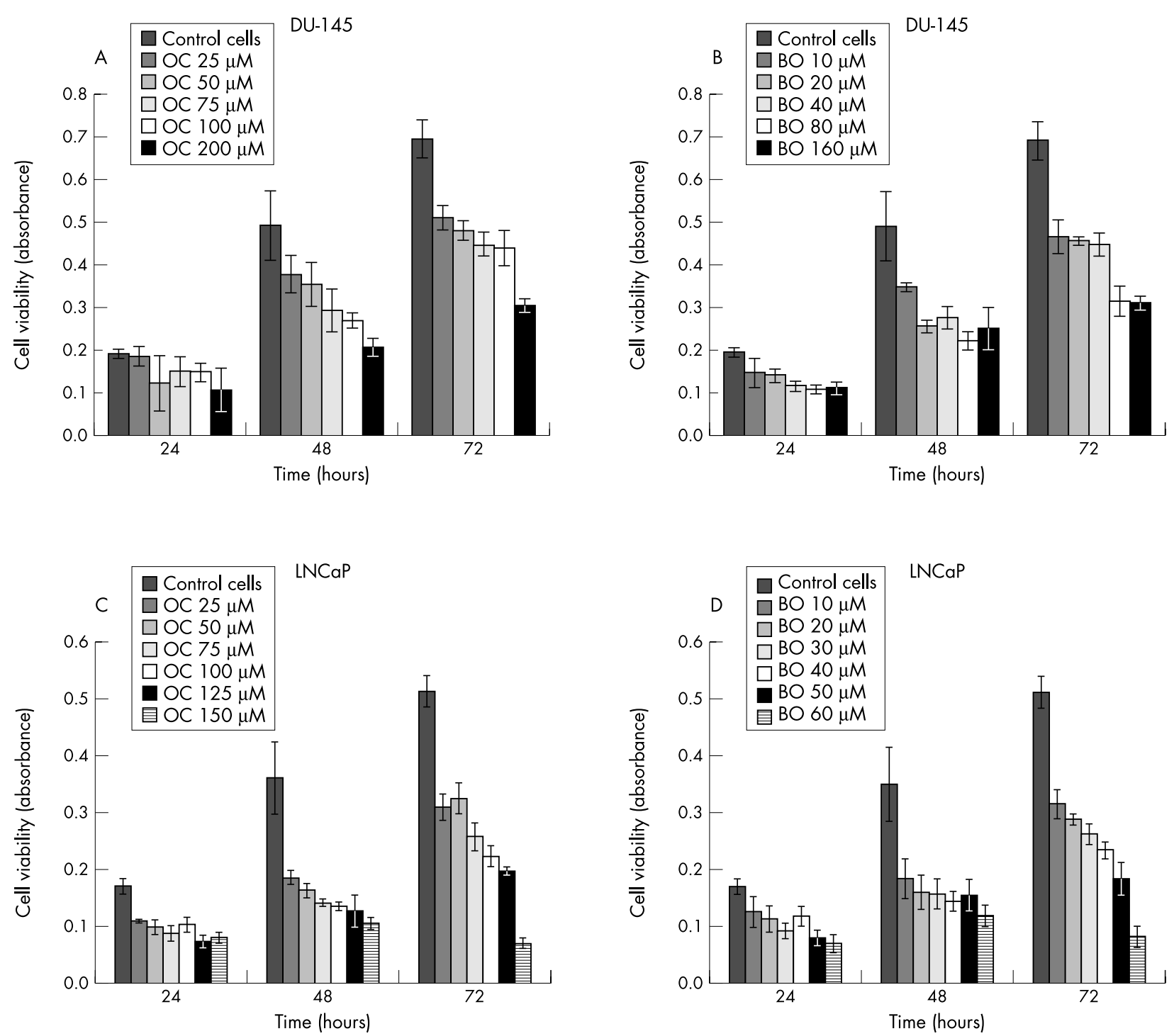

Figure 2 The effect of olomoucine $(\mathrm{OC})$ and bohemine $(\mathrm{BO})$ on cell viability measured by the MTT test. $(\mathrm{A})$ The effect of various concentrations of OC on DU-145 cells. (B) The effect of various concentrations of BO on DU-145 cells. (C) The effect of various concentrations of OC on LNCaP cells. (D) The effect of various concentrations of BO on LNCaP cells.

inhibition of cell growth; that is, $200 \mu \mathrm{M}$ olomoucine and

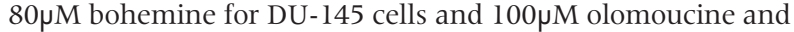

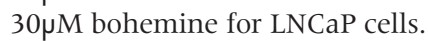

\section{Effect of CDK inhibitors on gene expression}

Figures 3 and 4 show the relative level of gene expression in both prostatic cell lines after treatment with olomoucine and bohemine. The expression of AR in the androgen sensitive LNCaP cell line was decreased after treatment for both 48 hours (olomoucine) and 72 hours (bohemine). In contrast, expression of AR in the androgen insensitive cell line DU-145 was increased.

Whereas Bax expression was increased in LNCaP cells after treatment (olomoucine was more efficient than bohemine), Bcl-2 expression was inhibited (with the exception of the effect of bohemine after 48 hours). In DU- 145 cells, where the bax gene is mutated, $\mathrm{Bcl}-2$ was not detectable and there was a tendency for Bax expression to decrease after treatment.

The effect of olomoucine and bohemine was different in cells with either wild-type (LNCaP) or defective p53 and pRB mediated (DU-145) regulatory pathways. Thus, the expression of p53 and p2 1 was rapidly and transiently induced in LNCaP but not in DU-145 cells. In fact, a reduction in the expression of both p53 and p21 was seen in DU- 145 cells after 48 and 72 hours. The expression of pRB and pl 6 was slightly increased in DU-145 cells but olomoucine and bohemine had no effect on the expression of these genes in LNCaP cells. In both cell lines, a double band for p16 was observed. The expression of p27 was increased in both cell lines.

In general, both olomoucine and bohemine inhibited the expression of cyclin A, cyclin B, and p34 ${ }^{\mathrm{cdc} 2}$ in both cell lines. The expression of cyclins Dl and E decreased in the DU-145 cells, whereas in LNCaP cells their expression was increased at both 24 and 48 hours after treatment. Western blot analysis for cyclin E revealed two bands in both the control and treated LNCaP cells, but the lower band was more intense in control untreated cells. When these cells were treated with olomoucine, after 24 and 48 hours there was a much more intensely stained upper band. These effects were not found after 72 hours of treatment with olomoucine or after treatment with bohemine.

\section{DISCUSSION}

The cell lines used in our study are the most frequently studied prostatic cancer cell lines. They have been well characterised by cytogenetics, mutation analysis, and protein 

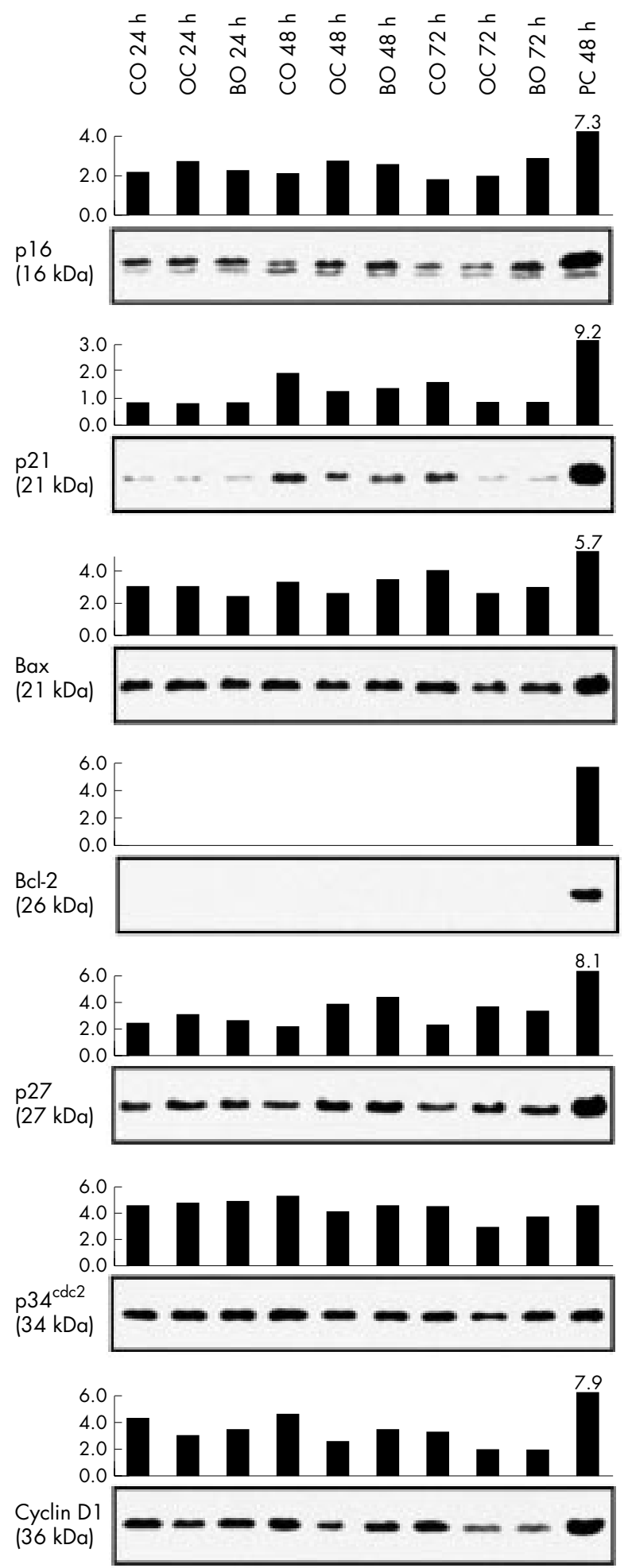
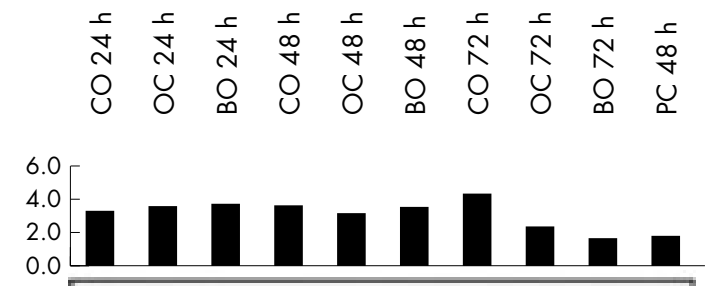

Cyclin E (50 kDa)
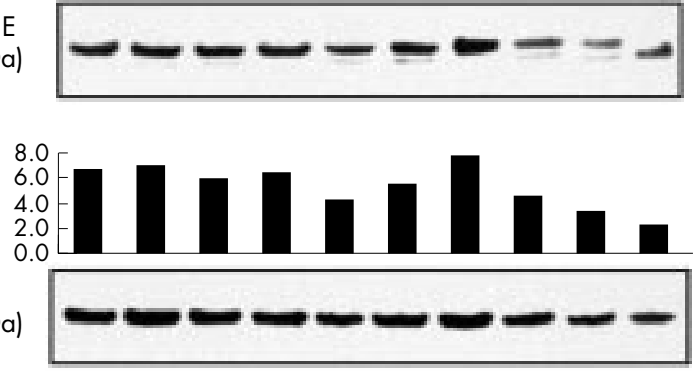

p53

$(53 \mathrm{kDa})$

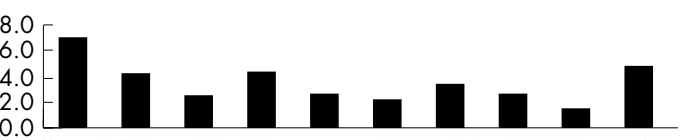

Cyclin A

$(60 \mathrm{kDa})$
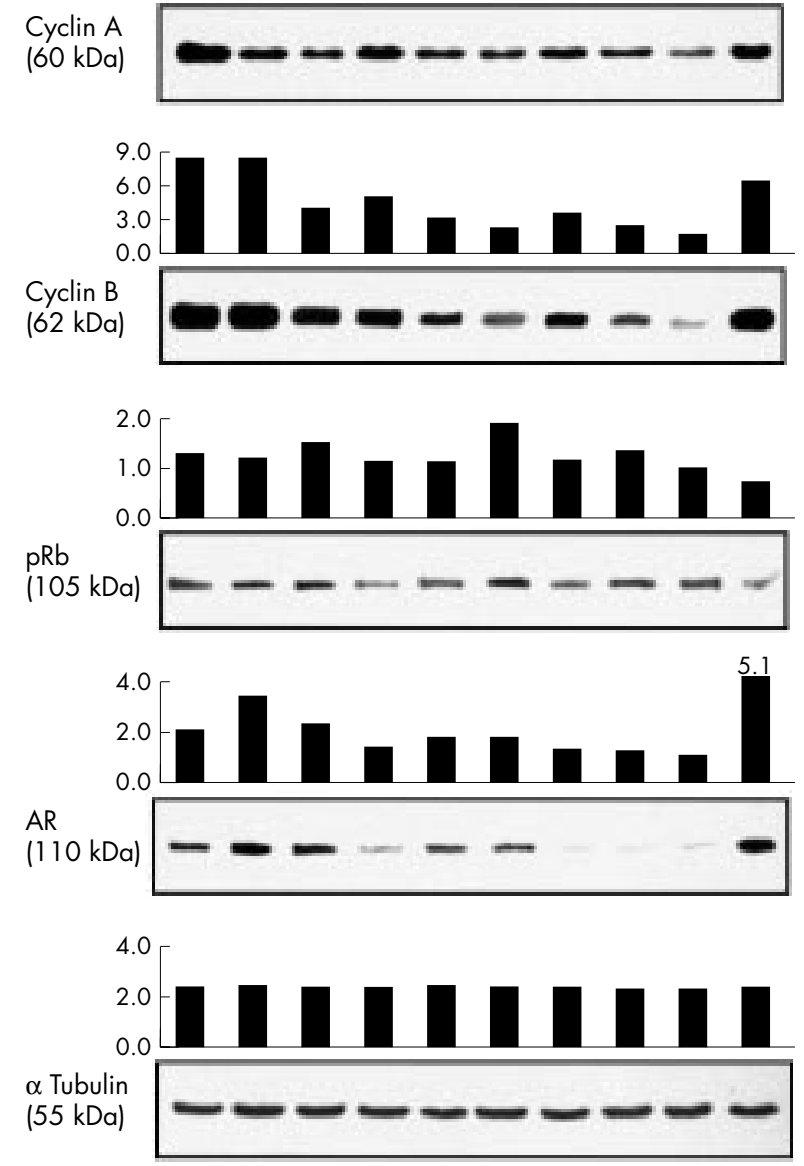

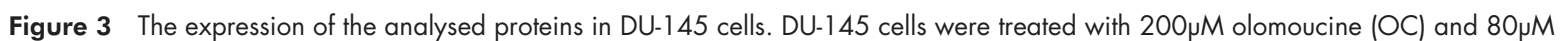
bohemine (BO) for 24, 48, and 72 hours. Cultures were sampled at these times for western blotting. The results were compared with expression in untreated control cells and in positive control cell lines (breast cancer cell lines MDA-MB-157 for p16, p34cdc2, and cyclin E, and MCF-7 for the remaining markers). The integral optical density of stained bands was determined using a GS-700 densitometer and Multi-analyst, version 1.1 software.

expression. ${ }^{15-18}$ The LNCaP cell line, originating from a prostatic tumour cell metastasised to a lymph node, contains only wild-type p53, carries a mutation in the hormone binding domain of AR, and expresses moderate amounts of androgen responsive AR. LNCaP has been used as a model for well differentiated, weakly tumorigenic, and non-metastatic (after subcutaneous application into nude mice) prostate cancer. The DU-145 cell line, derived from the brain metastasis of prostate cancer, is androgen insensitive, and carries a mutation in p53 and the bax gene. This line has been used as a model for poorly differentiated, aggressive, and metastatic prostatic cancer. DU-145 cells, in contrast to LNCaP cells, have been reported to 


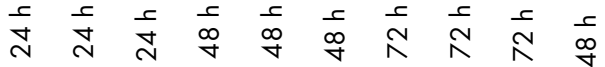

$$
\begin{aligned}
& \text { ○ U O }
\end{aligned}
$$
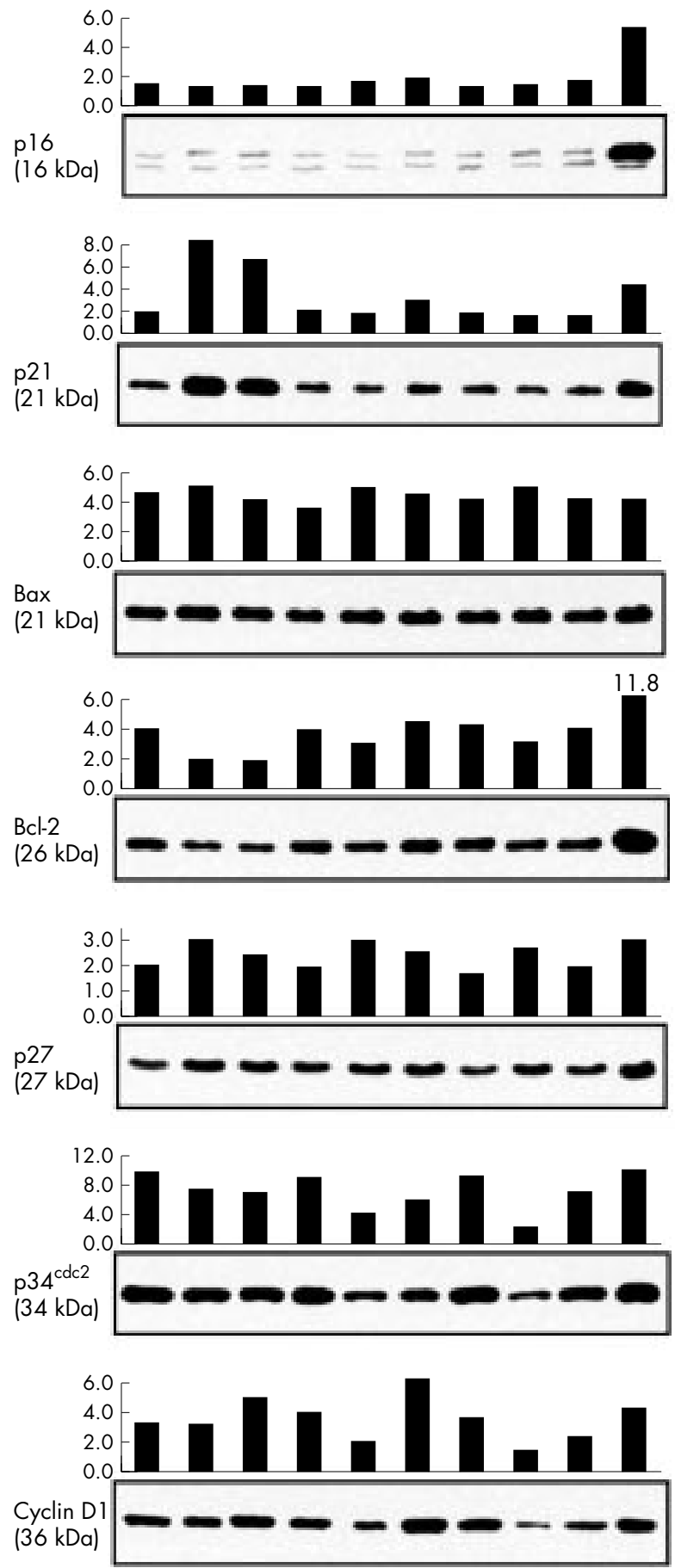
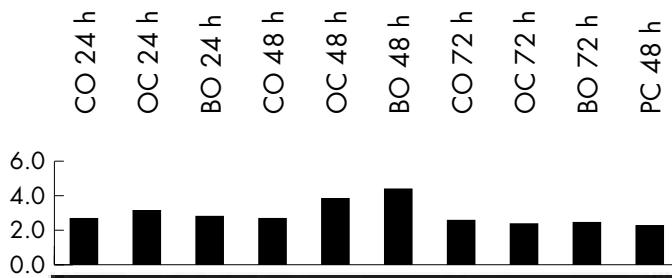

Cyclin E $(50 \mathrm{kDa})$
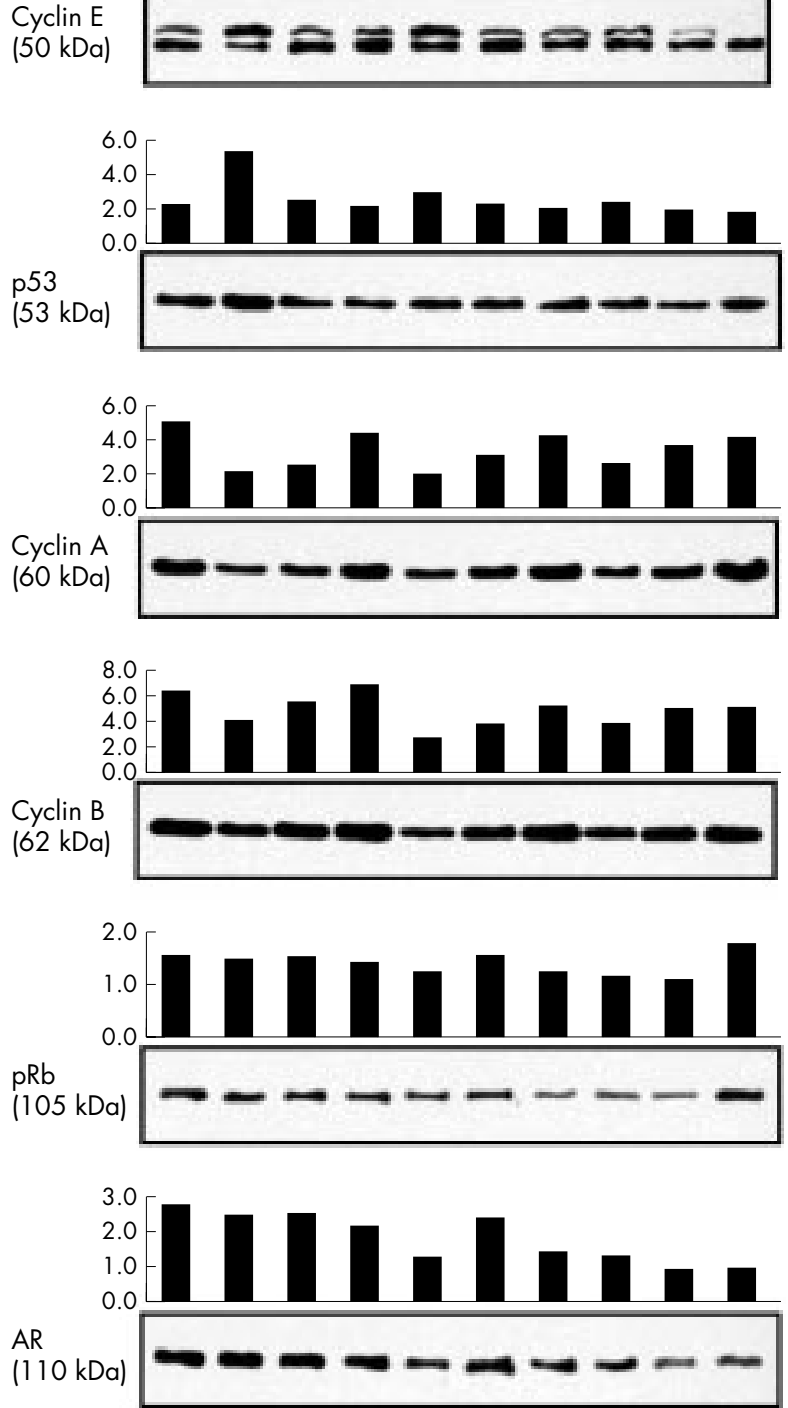

AR

$1110 \mathrm{kDa})$

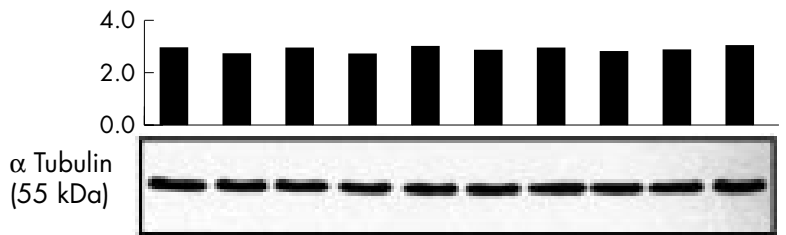

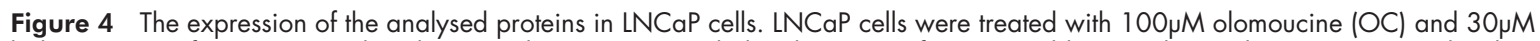
bohemine (BO) for 24, 48, and 72 hours. Cultures were sampled at these times for western blotting. The results were compared with expression in unaffected control cells and in positive control cell lines (breast cancer cell lines MDA-MB-157 for p16, p34cdc2, and cyclin E, and MCF-7 for the remaining markers). The integral optical density of stained bands was determined using a GS-700 densitometer and Multi-analyst, version 1.1 software

lack the AR protein and AR mRNA although there are no detectable alterations in the structure of the AR gene. ${ }^{19}$ Our repeated experiments, using two different monoclonal antibodies in western blot analysis, have shown that a low but detectable amount of the AR protein is present in the control DU-145 cells. Dorai and colleagues ${ }^{20}$ stated that LNCaP cells express only low amounts of Bcl-2, whereas Bcl-2 expression is undetectable in DU-145 cells. These findings are in agreement with our results.

The treatment of cells with olomoucine and bohemine stimulated changes in protein expression, which in our opinion correlate well with the changes in the expression of both 


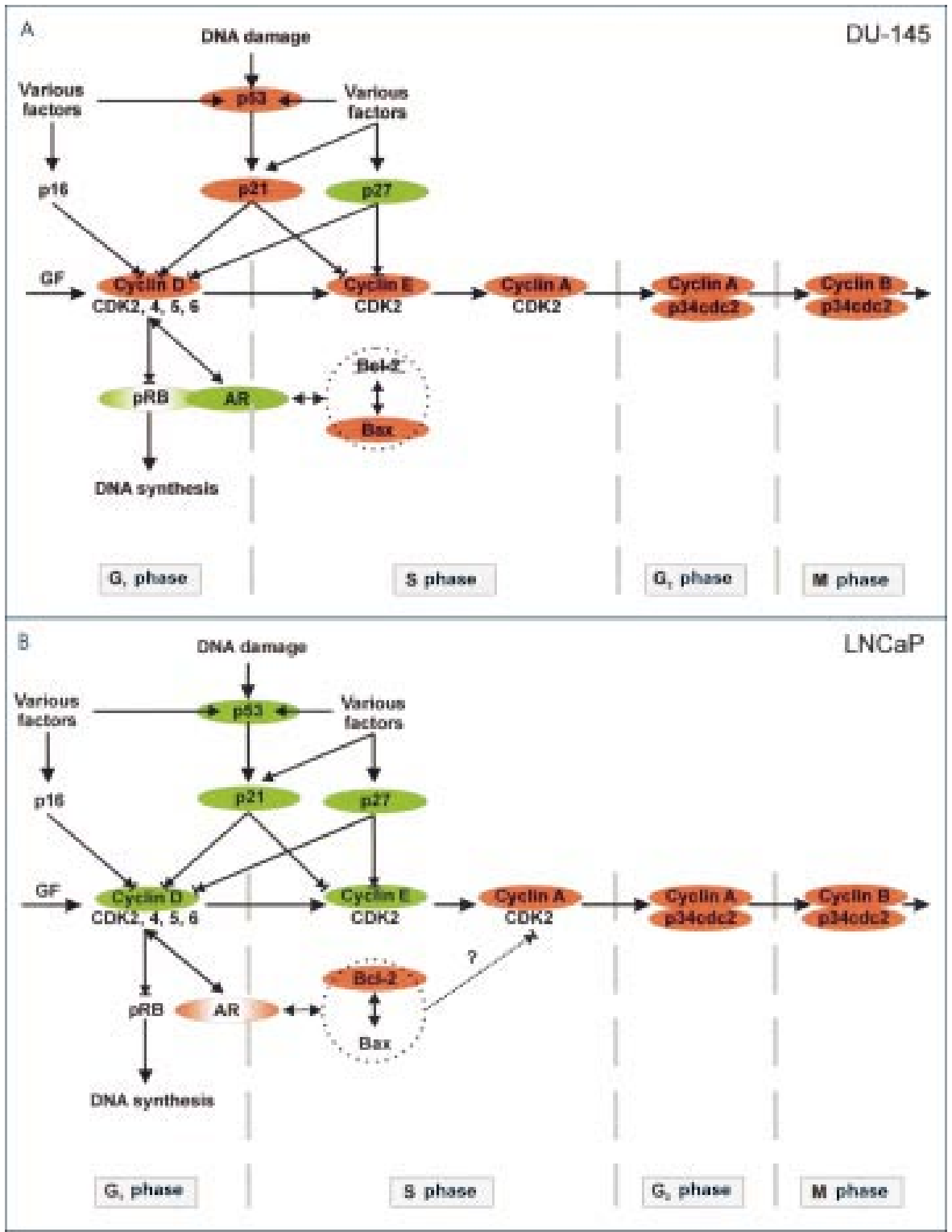

Figure 5 Scheme of presumed interactions of analysed markers during the cell cycle. (A) DU-145 cells. (B) LNCaP cells. Red colour, decreased expression; green colour, increased expression. AR, androgen receptor; CDK, cyclin dependent kinase; GF, growth factors; pRB, retinoblastoma protein.

AR and p53. The complexity of the regulatory pathways in the course of the cell cycle is schematically shown in fig 5A,B. The cells with wild-type p53 (LNCaP) are androgen sensitive, in spite of a mutation in the AR gene. They respond to treatment with CDK inhibitors by the classic pathway, with induction of p53 and the endogenous CDK inhibitors, p21 and p27. These changes are accompanied by a reduction in Bcl-2 expression (with the exception of an unexplained increase in Bcl-2 expression after 48 hours of bohemine treatment, accompanied by an increase in cyclin Dl and cyclin E) and a decrease in AR expression. In contrast, DU-145 cells carrying a mutation of p53 are androgen insensitive, probably because of a blockage of transcription of the normal AR gene. In addition, they are unable to regulate the cell cycle adequately because of the deleted RB gene and the mutated bax gene. Therefore, they respond to treatment by inhibition of the non-functional p53 mediated cascade, including p2l, and it is probable that they induce the aberrant pRB pathway. This may be explained by the modification of RB gene expression in the studied cell line and/or by the presence of a feedback mechanism causing the overexpression of non-functional pRB. These cells switch to another regulatory mechanism mediated by an increase in both AR and p27 values. We presume, in accordance with the findings of Tilley et al, that these ARs are functional and that they are able to regulate the growth of prostatic cancer cells. ${ }^{19}$ In spite of the reported close link between AR responsiveness and p27 induction in prostatic cancer, the inverse relation between p27 and AR expression in LNCaP cells after treatment with exogenous CDK inhibitors may reflect a relative independence of the p27 pathway to androgens in this cell line.

"The treatment of cells with olomoucine and bohemine stimulated changes in protein expression, which in our opinion correlate well with the changes in expression of both androgen receptor and p53"

In both cell lines the decreased expression of most cyclins and the CDK p34 $4^{\text {cdc2 }}$ after treatment with olomoucine and bohemine appears to be connected to the inhibitory action of these potent agents. However, the accumulation of cyclins D1 and $\mathrm{E}$ in LNCaP cells may indicate that inhibition of the cell 
cycle by synthetic CDK inhibitors is affected at various levels and phases (in DU-145 cells probably in Gl phase and in LNCaP cells in S phase), and that the site of their action may be dependent on the functional ability of various regulatory cascades. In LNCaP cells, two close bands were observed, probably corresponding to the isoforms of cyclin E, which may differ in their phosphorylation status. This finding may correspond to the upregulation of the cyclin E-CDK2 complex by regulators with phosphatase activity in cooperation with p27 and pRB, as has recently been suggested. ${ }^{21}$ Similar double bands of cyclin E were also found in DU-145 cells but the expression varied only in the upper band, whereas the lower band was only very weak and control untreated cells exhibited only one band in the upper position.

Our study showed that bohemine is two to three times more effective than olomoucine because this agent caused an approximately $50 \%$ inhibition of both proliferation (measured by estimation of DNA content) and viability (measured as the ability of metabolically active cells to convert tetrazolium salt) at lower concentrations. After application of both agents to DU-145 cells, the viability (metabolic activity of surviving cells) is constantly increased until 48/72 hours compared with 24 hours. This does not correspond to the finding of a lower number of cells as estimated by DNA content. One explanation could be that the surviving cells have a higher metabolic rate, as has been shown after treatment with various chemotherapeutics. ${ }^{22}$ A similar trend was also seen in LNCaP

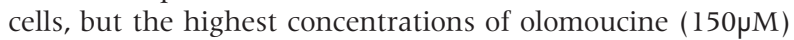
and bohemine $(60 \mu \mathrm{M})$ caused a stable inhibition of metabolism. This would be in agreement with the higher sensitivity of LNCaP cells to treatment. Similarly, the heterogenous response of different prostatic cancer cell lines to staurosporine treatment was recently described. ${ }^{23}$

"Our present study demonstrates the potential therapeutic benefit of synthetic cyclin dependent kinase inhibitors on both androgen sensitive and insensitive prostatic cancer cells"

Although the two agents used are related, their time dependent effects on protein expression differed. We found olomoucine to be a less potent inhibitor of the expression of cyclin A and B and a more potent inducer of AR expression in DU-145 cells than bohemine. Olomoucine was also able to induce more pronounced increases in p53, p21, p27, and Bax expression and decreases in cyclin D1, cyclin A, cyclin B, and AR expression in LNCaP cells. Bohemine, but not olomoucine, was a potent inducer of both cyclin Dl in LNCaP cells and pRB in DU-145 cells. These dissimilarities in action could result from a difference in their stability and susceptibility to degradation and/or a difference in their ability to induce cellular stress, which would agree with the recent finding of Rokhlin et al. ${ }^{24}$ Thus, differing expression of both AR and Bcl-2/Bax proteins seen in response to treatment could reflect a cellular effort to switch to survival pathways, which would depend on their gene equipment. A similar approach to the analysis of protein expression in prostate cell lines in the course of phenylbutyrate treatment was published recently. ${ }^{25}$ However, in that study the effect was accompanied by downregulation of Bcl-2 and by an increase in the ratio of Bax to Bcl-2, regardless of androgen dependency or p53 status. This finding documents the unique effect of both tested CDK inhibitors. In vitro, olomoucine and bohemine selectively inhibited members of the CDKl family of cyclin dependent kinases, such as CDKl, CDK2, CDK3, and CDK5, although there was no significant decrease in the activity of several major cellular kinases, including CDK4/6, mitogen activated protein kinase, protein kinase $\mathrm{C}$, and p21 activated protein kinase. ${ }^{26}$ However, recently we have shown that olomoucine derivatives are potent inhibitors of CDK4 and CDK7 in vivo. ${ }^{27}$ The cellular

\section{Take home messages}

- Olomoucine and bohemine are both potent inhibitors of growth and viability, although bohemine is two to three times more effective than olomoucine

- The sensitivity of the androgen sensitive LNCaP cells to both agents is significantly higher than that seen in the androgen insensitive DU-145 cells

- Treatment with these two cyclin dependent kinase inhibitors had secondary effects on the expression of numerous regulators, so that these agents might modulate the responsiveness of tumour cells to treatment, including treatment with hormone antagonists

effects of olomoucine were investigated in a large variety of plant and animal models. ${ }^{38}$ The growth of tumour cells was inhibited by olomoucine and the related agent, roscovitine, and both compounds were able to inhibit the cell cycle at Gl/S and G2/M transitions ${ }^{26}{ }^{29}{ }^{30}$ Although olomoucine is a relatively weak CDK inhibitor, we have shown that this drug has sufficient efficacy to induce apoptosis and tumour regression after in vivo application in several animal tumour models and in the case of spontaneous dog malignant melanoma. ${ }^{31}$

Our present study demonstrates the potential therapeutic benefit of synthetic CDK inhibitors on both androgen sensitive and insensitive prostatic cancer cells. Nevertheless, their effect on the course of the cell cycle and the survival of cancer cells is quite different and depends on the pool of genes involved in both lines. As can be seen from fig 5A,B, LNCaP and DU-145 cells are inhibited in different phase of the cell cycle. Apart from their direct inhibitory effect on the cell cycle, they also exhibit valuable secondary effects on the expression of upstream or downstream regulators, and thus may modulate the responsiveness of tumour cells to treatment. These agents can also activate the expression of ARs in at least some of the androgen insensitive cancer cells, which thus allows us to speculate about the presensitisation of androgen insensitive prostate cancer cells before treatment with hormone antagonists.

\section{ACKNOWLEDGEMENT}

This work was supported by grant NC 6200-3.

\section{Authors' affiliations}

J Mad'arová, M Lukešová, A Hlobilková, Z Kolář, Laboratory of Molecular Pathology, Faculty of Medicine, Centre of Molecular Biology and Medicine, Palacký University, Hněvotínská 3, CZ-77515 Olomouc, Czech Republic

M Strnad, B Vojtěšek, $\mathbf{R}$ Lenobel Laboratory of Growth Regulators, Faculty of Science, Centre of Molecular Biology and Medicine, Palacký University, CZ-78371, Olomouc, Czech Republic

M Hajdúch, Laboratory of Experimental Medicine, Faculty of Medicine and Faculty Hospital, Centre of Molecular Biology and Medicine, Palacký University, CZ-77520, Olomouc, Czech Republic

P G Murray, CRC Institute for Cancer Studies, University of Birmingham, Birmingham B15 2TJ, UK

S Perera, Biomedical Science Research Laboratories, School of Health

Sciences, University of Wolverhampton, Wolverhampton WVI 1DJ, UK

\section{REFERENCES}

1 Havlíček L, Hanuš J, Veselý J, et al. Cyłokinin-derived cyclin-dependent kinase inhibitors: synthesis and cdc2 inhibitory activity of olomoucine and related compounds. J Med Chem 1997;40:408-12.

2 Garrett MD, Fattaey A. CDK inhibition and cancer therapy. Current Opin Genet Dev 1999;9:104-11.

3 Abraham RT, Acquarone M, Andersen A, et al. Cellular effects of olomoucine, an inhibitor of cyclin-dependent kinases. Biol Cell 1995;83:105-20.

4 Meijer L, Bisagni E, Legraverend $M$, et al. Novel purine derivatives having, in particular, antiproliferative properties, and biological uses thereof. Organisation mondiale de la propriete intellectuelle. 1997;WO $97 / 20842$ 
5 Schuurmans ALG, Bolt J, Veldscholte J, et al. Regulation of growth of LNCaP human prostate tumor cells by growth factors and steroid hormones. Steroid Biochem Mol Biol 1991;40:193-7.

6 Dorkin TJ, Neal DE. Basic science aspects of prostate cancer. Semin Cancer Biol 1997:8:21-7.

7 Apakama I, Robinson MC, Walter NM, et al. Bcl-2 overexpression combined with p53 protein accumulation correlates with hormone-refractory prostate cancer. Br J Cancer 1996;74: 1258-62.

8 Magi-Galluzzi C, Murphy M, Cangi G et al. Proliferation, apoptosis and cell cycle regulation in prostatic carcinogenesis. Quant Cytol Histol 1998:20:343-50

9 Bai XZ, Masters JR, O'Donoghue N, et al. Prognostic markers in clinically localised prostate cancer. Int J Pathol 1999; 14:785-91.

10 Klocker H, Culig Z, Hobish A, et al. Androgen receptor alterations in prostatic carcinoma. Prostate 1994;25:266-73.

11 Rao J, Otto WR. Fluorimetric DNA assay for cell growth estimation. Anal Biochem 1992;207:186-92.

12 Alley MC, Scudieco DA, Monks A, et al. Feasibility of drug screening with panels of human tumor cell lines using a microculture tetrazolium assay. Cancer Res 1988;48:589-601.

13 Roehm NW, Rodgers GH, Hatfield SM, et al. An improved colorimetric assay for cell proliferation and viability utilizing the tetrazolium salt XTT. Immunol Methods 1991;142:257-65.

14 Lee VH, Dunbar BS. Sample preparation for protein electrophoresis and transfer. In: Dunbar BS, ed. Protein blotting: a practical approach. Oxford: Oxford University Press, 1994:87-101.

15 Carroll AG, Voeller HJ, Surgars L, et al. p53 oncogene mutations in the three human prostate cancer cell lines. Prostate 1993;23:123-4.

16 Rampino $\mathbf{N}$, Yamamoto $\mathrm{H}$, lonov $\mathrm{Y}$, et al. Somatic frameshift mutations in the BAX gene in colon cancers of the microsatellite mutator phenotype. Science 1997; 275:967-9.

17 Beham AW, Sarkiss M, Brisbay S, et al. Molecular correlates of bcl-2-enhanced growth following androgen-ablation in prostate carcinoma cell in vivo. Int J Mol Med 1998;1:953-9.

18 Fan S, Wang J-A, Yuan R, et al. BRCAl as a potential human prostate tumor suppressor: modulation of proliferation, damage responses and expression of cell regulatory proteins. Oncogene 1998;16:3069-82.

19 Tilley WD, Wilson CM, Marcelli M, et al. Androgen receptor gene expression in human prostate carcinoma cell lines. Cancer Res 1990;50:5382-6.
20 Dorai T, Perlman $\mathrm{H}$, Walsh $\mathrm{K}$, et al. A recombinant defective adenoviral agent expressing anti-bcl-2 ribozyme promotes apoptosis of bcl-2-expressing human prostate cancer cells. Int J Cancer 1999;82:846-52.

21 Cheney IW, Neuteboom STC, Vaillancourt M-T, et al. Adenovirus-mediated gene transfer of $\mathrm{MMACl}$ /PTEN to glioblastoma cells inhibits $S$ phase entry by the recruitment of $\mathrm{p} 27 \mathrm{Kip} 1$ into cyclin E/CDK2 complexes. Cancer Res 1999:59:2318-23.

22 Elgie AW, Sargent JM, Taylor CG, et al. An in vitro study of blast cell metabolism in acute myeloid leukaemia using MTT assay. Leuk Res 1996;20:407-13

23 Marcelli M, Marani M, Li X, et al. Heterogeneous apoptotic responses of prostate cancer cell lines identify an association between sensitivity to staurosporine-induced apoptosis, expression of $\mathrm{Bcl}-2$ family members, and caspase activation. Prostate 2000:42:260-73

24 Rokhlin OW, Gudkov AV, Kwek S, et al. p53 is involved in tumor necrosis factor- $\alpha$-induced apoptosis in the human prostatic carcinoma cell line LNCaP. Oncogene 2000;19:1959-68

$25 \mathrm{Ng}$ AY, Bales W, Veltri RW. Phenylbutyrate-induced apoptosis and differential expression of $\mathrm{Bcl}-2, \mathrm{Bax}, \mathrm{p} 53$ and $\mathrm{Fas}$ in human prostate cancer cell lines. Anal Quant Cytol Histol 2000;22:45-54.

26 Meijer L, Borgne A, Mulner O, et al. Biochemical and cellular effects of roscovitine, a potent and selective inhibitor of the cyclin-dependent kinases cdc2, cdk2 and cdk5. Eur J Biochem 1997;243:527-36.

27 Hajdúch $M$, Nosková V, Feketová G, et al. Olomoucine derived synthetic cyclin dependent kinase inhibitors - new generation of potent anti-cancer drugs. In: Pieters R, Veerman A, Kaspers GJL, eds. Drug resistance in leukaemia and lymphoma, advances in experimental medicine and biology. New York: Kluwer Academic/Plenum Press, 1999:341-53.

28 Alberio R, Kubelka M, Zakhartchenko V, et al. Activation of bovine oocytes by specific inhibition of cyclin-dependent kinases. Mol Reprod Dev 2000;55:422-32.

29 Veselý J, Havlíček L, Strnad M, et al. Inhibition of cyclin-dependent kinases by purine analogues. Eur J Biochem 1994;224:771-86.

30 Schutte B, Nieland L, van Engeland $M$, et al. The effect of the cyclin-dependent kinase inhibitor olomoucine on cell cycle kinetics. Exp Cell Res 1998;236:4-15

31 Hajdúch M, Koláŕ Z, Novotný R, et al. Induction of apoptosis and regression of spontaneous dog melanoma following in vivo application of synthetic cyclin dependent kinase inhibitor olomoucine. Anticancer Drugs 1997:8:1007-10.

\section{$\mathrm{ECHO}$}

\section{p21: prognostic marker in pleural mesothelioma}
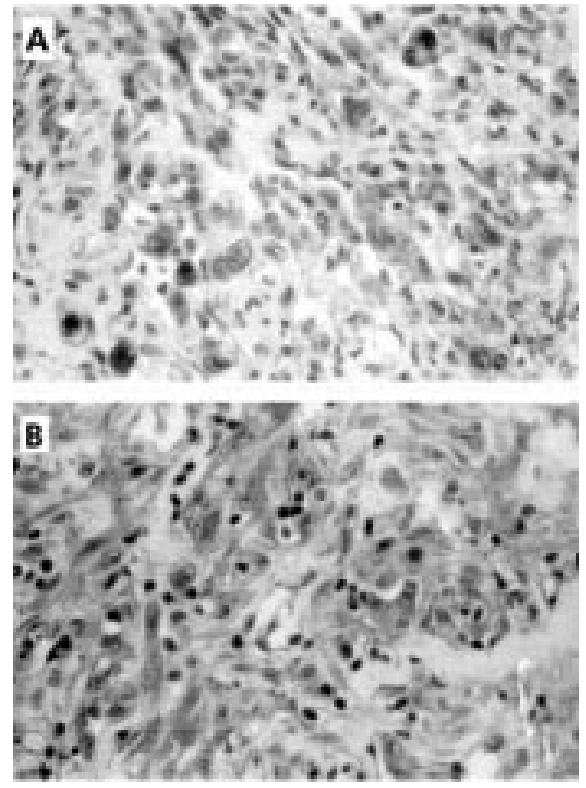

Mesothelioma stained with monoclonal antibody to $\mathrm{p} 21$. (A) Mixed mesothelioma with $\leqslant 10 \%$ of positive cells; (B) sarcomatoid mesothelioma with $>10 \%$ positive cells. Original magnification $\times 400$.
Expression of p2 1 -an inhibitor of the cell cycle-may hold an important clue to events - leading to malignant mesothelioma. Normally p21 expression is upregulated by the binding of p53 to the p21 promoter, but in malignant mesothelioma-an importan cancer of the pleura-it may be silenced by the binding and inactivation of $\mathrm{p} 53$ by simian virus 40 large $\mathrm{T}$ antigen (SV40Tag). This preliminary study supports the hypothysis.

Expression of $\mathrm{p} 2 \mathrm{l}$ in specimens of malignant mesothelioma was not significantly related to histological type in the study. However, median survival in the patients was significantly greater for specimens expressing p 21 in more than $10 \%$ of cells (median difference in survival 7 months, $95 \%$ confidence interval 4.8 to 9.9 months), and Kaplan-Meier analysis confirmed the relation.

Twenty nine specimens of malignant mesothelioma were assessed-all known to be positive for SV40Tag. Sixteen tumours were epithelial, six sarcomatoid, and seven were mixed tumours. p2 l expression was determined with a specially developed immunohistochemical method capable of discriminating non-cancerous cell types (stroma and endothelial and inflammatory cells). Survival data were obtained from patients' notes or interviews with relatives and excluded two deaths from causes unrelated to mesothelioma.

Malignant mesothelioma is increasing. It is not always caused by asbestos, and the exact mechanism of pathogenesis is unknown, though recently SV40Tag has been implicated through binding to $\mathrm{p} 53$. As a downstream target of p53, p21 is a potential candidate for studying the molecular mechanisms of pathogenesis.

A Thorax 2002;57:353-356.

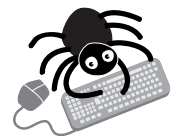

Please visit the Molecular Pathology website [www.molpath.com] for link to this full article. 